\title{
Odd-frequency superconductivity near a magnetic impurity in a conventional superconductor
}

\author{
Dushko Kuzmanovski $\odot,{ }^{1}$ Rubén Seoane Souto $\odot,{ }^{2,3}$ and Alexander V. Balatsky ${ }^{1,4}$ \\ ${ }^{1}$ Nordita KTH Royal Institute of Technology and Stockholm University SE-106 91 Stockholm, Sweden \\ ${ }^{2}$ Division of Solid State Physics and NanoLund, Lund University, S-22100 Lund, Sweden \\ ${ }^{3}$ Center for Quantum Devices and Station Q Copenhagen, Niels Bohr Institute, University of Copenhagen, DK-2100 Copenhagen, Denmark \\ ${ }^{4}$ Department of Physics, University of Connecticut, Storrs, Connecticut 06269, USA
}

(Received 19 December 2019; revised manuscript received 7 February 2020; accepted 10 February 2020; published 4 March 2020)

\begin{abstract}
Superconductor-ferromagnetic heterostructures have been suggested as one of the most promising alternatives of realizing odd-frequency superconductivity. In this work we consider the limit of shrinking the ferromagnetic region to the limit of a single impurity embedded in a conventional superconductor, which gives rise to localized Yu-Shiba-Rusinov (YSR) bound states with energies inside the superconducting gap. We demonstrate that all the sufficient ingredients for generating odd-frequency pairing are present at the vicinity of these impurities. We investigate the appearance of all possible pair amplitudes in accordance with the Berezinskii $S P^{*} O T^{*}=-1$ rule, with the symmetry under the exchange of spin, spatial, orbital (in our case $O=+1$ ), and time index, respectively. We study the spatial and frequency dependence of the possible pairing amplitudes, analyzing their evolution with impurity strength and identifying a reciprocity between different symmetries related through impurity scattering. We show that the odd-frequency spin-triplet pairing amplitude dominates at the critical impurity strength, where the YSR states merge at the middle of the gap, while the even components are quenched close to the impurity. We also show that the spin-polarized local density of states exhibits the same spatial and frequency behavior as the odd- $\omega$ spin-triplet component at the critical impurity strength.
\end{abstract}

DOI: 10.1103/PhysRevB.101.094505

\section{INTRODUCTION}

Odd-frequency (odd- $\omega$ ) superconducting (SC) pairing is a proposed unconventional dynamic SC state that is both nonlocal and odd in the relative time coordinate [1]. Berezinskii was the first to point out that the only requirement on the pair correlator is to be antisymmetric under a simultaneous exchange of spin $(S)$, spatial $\left(P^{*}\right)$, and time $\left(T^{*}\right)$ labels [1,2], which was later extended for multiorbital (multiband) systems [3] to include exchange of the orbital index $(O)$. It is written concisely as $S P^{*} O T^{*}=-1$ and referred to as Berezinskii rule. By allowing for odd time (or, equivalently, frequency) dependence, the two possible symmetries (spin-singlet evenparity and spin-triplet odd-parity) were extended to two more (spin-singlet odd-parity and spin-triplet even-parity) [4]. The odd- $\omega$ spin-triplet and even-parity order was initially proposed in liquid ${ }^{3} \mathrm{He}$ and referred to as Berzinskii state. It was eventually ruled out in favor of the even-frequency, spin-triplet, odd-parity [5,6]. This idea drove the interest on realizing odd- $\omega$ states in solids. Later, it has been shown that phonon-mediated electron-electron interactions cannot stabilize an odd- $\omega$ SC order parameter (OP) [7], but other mechanisms based on spin-fluctuation-mediated interactions

Published by the American Physical Society under the terms of the Creative Commons Attribution 4.0 International license. Further distribution of this work must maintain attribution to the author(s) and the published article's title, journal citation, and DOI. Funded by Bibsam. dependent on the Cooper pairs spin may be favorable [8,9]. Several systems, such as disordered systems, [10-12] heavy fermion and Kondo systems [13-16], and, more recently, Dirac semimetals [17], were contemplated as theoretical possibilities of stabilizing an odd- $\omega$ SC OP. These materials were shown to exhibit exotic electromagnetic properties $[18,19]$.

A different approach to inducing odd- $\omega$ pair correlations is through engineering heterostructures where a conventional (even-frequency, spin-singlet, $s$-wave) SC is proximitized to region that causes the breaking of some of its symmetries. This generates a corresponding odd- $\omega$ correlation component (see Sec. IC in Ref. [4]). Historically the first and the most analyzed systems are the superconductor-ferromagnet (SF) heterojunctions where the odd- $\omega$ spin-triplet $s$-wave pair correlations survive disorder [20,21]. Issues concerning various geometries, magnetization profiles and the effects on different proximity and inverse proximity induced orders were considered (for a recent review see Ref. [22]). More recently, the interest in these heterojunctions was revived in relation to their potential application in superconducting spintronic devices [23-26].

Conceptually, the sufficient ingredients for generating odd$\omega$ pair correlations by proximity effect may be preserved while transitioning from the geometry of heterostrutures, through finite-sized ferromagnetic islands to the limit of an isolated magnetic impurity, as illustrated in Fig. 1. In this work we consider the extreme case of reducing the size of the ferromagnetic island, which corresponds to the controlled immersion of a single magnetic impurity atom in a clean superconductor in Fig. 1(c). 

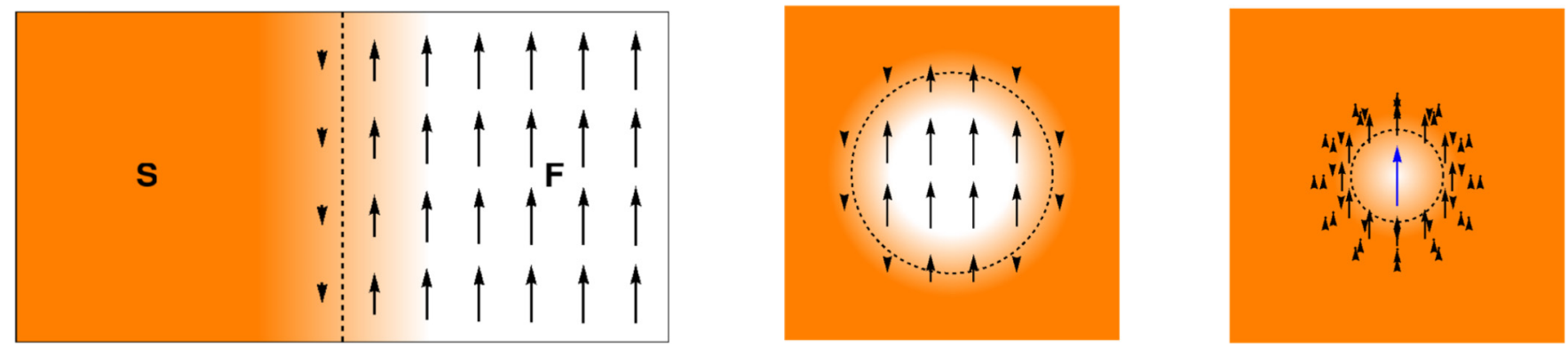

FIG. 1. The conceptual decrease of the size of the magnetic region: (a) an SF heterojunction. The orange color's saturation represents the magnitude of the conventional pair correlations, encoding the proximity effect in the ferromagnetic (F) region, and the inverse proximity effect in the $\mathrm{S}$ region. The arrow length is the magnitude of the local magnetization, leaking in the $\mathrm{S}$ region; (b) a ferromagnetic island inside a bulk SC; and (c) a single magnetic impurity immersed in a bulk SC. The blue arrow is the fixed magnetic moment of the impurity.

These magnetic impurities were instrumental in mapping out the spatial symmetry of the order parameter through scanning tunneling spectroscopic measurements in the cuprate superconductors [27]. A well-known result [28-30] is the appearance of spin-polarized subgap Yu-Shiba-Rusinov (YSR) bound states localized around the impurity position, whose energy can cross zero as the impurity strength is varied. A disordered dense collection of such impurities causes a filling of the energy gap and suppression of bulk superconductivity. There has been a renewed experimental [31-34] and theoretical [35-49] interest in magnetic impurities on superconductors, mostly due to the possibility of a realization of a one-dimensional topological superconductor that host zero-energy Majorana end modes in the presence of spin-orbit coupling.

In this work, we consider the limiting situation of a magnetic defect [Fig. 1(c)], namely an isolated magnetic impurity atom immersed in a bulk conventional SC. Previous works have shown that a spin symmetry breaking mechanism, such as a magnetic field or the coupling to ferromagnetic electrodes can induce odd- $\omega$ pair correlations [50,51]. Here we demonstrate that a magnetic impurity is sufficient to generate these pair correlations, in accordance with the $S P^{*} O T^{*}$ rule. Very recently, Ref. [52] a complementary study has shown the existence of odd- $\omega$ in nonmagnetic potential impurities due to the renormalization of the SC OP. This corresponds to the limiting case of a metal-superconductor (NS) heterojunctions (where the $\mathrm{N}$ region has been reduced to an impurity), shown to to host odd- $\omega$ pair correlations when the spatial modulation of the SC OP is taken into account [53-55].

The simple geometry under consideration offers the advantage of exactly decomposing the spin, spatial, and frequency dependence of the pair correlations, enabling a clear demonstration of the conversion of spin and time symmetry. Motivated by the experimental capabilities in measuring magnetic impurities in superconductors [56,57], it is worthwhile to point out the features of the odd- $\omega$ Berezinskii pairing that are correlated with spectroscopic data [58-61]. We discuss the behavior of the spin-polarized (SP) local density of states (LDOS) as it can be related to the pair correlations.

The rest of the article is organized as follows: In Sec. II we describe the model Hamiltonian of the system formed by a Kondo-type impurity immersed in a BCS bulk SC. We introduce the Green's function and the Dyson equation in
Sec. II A. The results are presented in Sec. III. In Sec. III A, we provide analytical expressions for the impurity Green's function. In Sec. III B, the reciprocity relations between the pair correlation components are introduced, discussing the parameters where odd- $\omega$ components dominate. In Sec. III C we show the SP LDOS close to the impurity and local magnetization DOS (LMDOS). In Secs. IIID and IIIE the pair correlations are represented. Finally, analytic expressions and derivation details are given in the Appendices.

\section{MODEL AND METHOD}

We consider a clean metal with spin-degenerate and isotropic dispersion $\xi_{\mathbf{k}} \approx v_{F}\left(|\mathbf{k}|-k_{F}\right)$ in $D$ spatial dimensions whose Fermi surface is a sphere of radius $k_{F}$ and the band velocity at the Fermi level (Fermi velocity) is $v_{F}$. The metal is an intrinsic conventional $\mathrm{SC}$, with a spatially uniform [62] singlet $s$-wave SC OP $\Delta$. The Hamiltonian of the system is given by $\mathcal{H}=\mathcal{H}_{\mathrm{BCS}}+\mathcal{H}_{\text {imp }}$, where the metal is a $\mathrm{BCS}$ mean-field Hamiltonian:

$$
\mathcal{H}_{\mathrm{BCS}}=\sum_{\mathbf{k} \sigma} \xi_{\mathbf{k}} c_{\mathbf{k} \sigma}^{\dagger} c_{\mathbf{k} \sigma}+\sum_{\mathbf{k}}\left\{\Delta c_{\mathbf{k} \uparrow}^{\dagger} c_{-\mathbf{k} \downarrow}^{\dagger}+\text { H.c. }\right\}
$$

Here $c_{\mathbf{k} \sigma}\left(c_{\mathbf{k} \sigma}^{\dagger}\right)$ is the second-quantized electron annihilation (creation) operator that destroys (creates) an electron with wave vector $\mathbf{k}$ and spin projection $\sigma \in\{\uparrow, \downarrow\}$.

Inside the metal, a pointlike magnetic impurity at position $\mathbf{x}=\mathbf{0}$ is immersed. The impurity Hamiltonian is a Kondo-type Hamiltonian described by

$$
\mathcal{H}_{\text {imp }}=U_{m}[\mathbf{n} \cdot \mathbf{M}(0)],
$$

where $U_{m}$ is the impurity strength and $\mathbf{n}$ is a unit vector $[(\mathbf{n} \cdot \mathbf{n})=1]$ that determines the polarization of the magnetic impurity. $\mathbf{M}(\mathbf{x})$ is the real-space spin polarization due to the conduction electrons:

$$
M_{i}(\mathbf{x})=\sum_{\sigma \sigma^{\prime}}\left[\sigma_{i}\right]_{\sigma \sigma^{\prime}} c_{\sigma}^{\dagger}(\mathbf{x}) c_{\sigma^{\prime}}(\mathbf{x}),
$$

with $i=(x, y, z)$ being a Cartesian component label. Here $c_{\sigma}^{\dagger}(\mathbf{x})$ is a creation operator that creates an electron at position $\mathbf{x}$ with spin polarization $\sigma$. It is related to the 
momentum-space via a Fourier transform:

$$
c_{\sigma}^{\dagger}(\mathbf{x})=\frac{1}{L^{D / 2}} \sum_{\mathbf{k}} e^{-i \mathbf{k} \cdot \mathbf{x}} c_{\mathbf{k} \sigma}^{\dagger},
$$

and analogously for $c_{\sigma}(\mathbf{x})$. The minus sign in the exponent is in accordance with the choice for the translation operator $T(\mathbf{a})=\exp [-i(\mathbf{a} \cdot \mathbf{P})]$ (we choose units in which $\hbar=k_{B}=$ 1) so that $T(\mathbf{a}) c_{\sigma}^{\dagger}(\mathbf{x}) T^{\dagger}(\mathbf{a})=c_{\sigma}^{\dagger}(\mathbf{x}+\mathbf{a})$. The normalization $L^{-D / 2}$ ensures that the real-space second-quantized operators satisfy the anticommutation relation:

$$
\left\{c_{\sigma}(\mathbf{x}), c_{\sigma^{\prime}}^{\dagger}\left(\mathbf{x}^{\prime}\right)\right\}=\delta_{\sigma \sigma^{\prime}} \delta^{D}\left(\mathbf{x}-\mathbf{x}^{\prime}\right) .
$$

Thus $\left[c_{\sigma}^{\dagger}(\mathbf{x})\right]=\left[c_{\sigma}(\mathbf{x})\right]=L^{-D / 2}$, and $[M]=L^{-D}$. In order that $\mathcal{H}_{\text {imp }}$ has dimension of energy, $U_{m}$ ought to have dimension $\left[U_{m}\right]=\mathrm{L}^{D}[E]$.

We define the Nambu spinor:

$$
\Psi(\mathbf{x})=\left(c_{\sigma}(\mathbf{x}), \quad\left(i \sigma_{y}\right)_{\sigma \sigma^{\prime}} c_{\sigma^{\prime}}^{\dagger}(\mathbf{x})\right)^{\top},
$$

which satisfies the commutation relations with the spin operator:

$$
-\left[S_{a}, \Psi(\mathbf{x})\right]=\frac{1}{2}\left(\hat{\tau}_{0} \otimes \hat{\sigma}_{a}\right) \cdot \Psi(\mathbf{x}),
$$

where the Pauli matrices $\hat{\tau}_{\mu}$ and $\hat{\sigma}_{\mu}$ operate in the Nambu and the spin subspaces, respectively. We define an imaginarytime-ordered Green's function matrix:

$$
\begin{aligned}
\check{G}\left(\mathbf{x}, \tau ; \mathbf{x}^{\prime}, \tau^{\prime}\right) & =-\left\langle T_{\tau} \Psi(\mathbf{x}, \tau) \otimes \bar{\Psi}\left(\mathbf{x}^{\prime}, \tau^{\prime}\right)\right\rangle \\
& =\left(\begin{array}{c|c}
\hat{G}^{e e}\left(\mathbf{x}, \tau ; \mathbf{x}^{\prime}, \tau^{\prime}\right) & \hat{G}^{e h}\left(\mathbf{x}, \tau ; \mathbf{x}^{\prime}, \tau^{\prime}\right) \\
\hat{G}^{h e}\left(\mathbf{x}, \tau ; \mathbf{x}^{\prime}, \tau^{\prime}\right) & \hat{G}^{h h}\left(\mathbf{x}, \tau ; \mathbf{x}^{\prime}, \tau^{\prime}\right)
\end{array}\right),
\end{aligned}
$$

where ${ }^{`}$ denotes a $4 \times 4$ matrix in the Nambu-spin space. The imaginary-time Heisenberg operators evolve with the total Hamiltonian of the system $\mathcal{H}$ according to:

$$
\begin{aligned}
& \Psi(\mathbf{x}, \tau)=e^{\tau \mathcal{H}} \Psi(\mathbf{x}) e^{-\tau \mathcal{H}}, \\
& \bar{\Psi}(\mathbf{x}, \tau)=e^{\tau \mathcal{H}} \Psi^{\dagger}(\mathbf{x}) e^{-\tau \mathcal{H}} .
\end{aligned}
$$

In case of a time-independent Hamiltonian, the Green's function Eq. (8) is only a function of the time difference $\tau-\tau^{\prime}$ and may be expanded in fermionic Matsubara frequencies:

$$
\check{G}\left(\mathbf{x}, \tau ; \mathbf{x}^{\prime}, \tau^{\prime}\right)=\frac{1}{\beta} \sum_{\omega_{m}} e^{-i \omega_{m}\left(\tau-\tau^{\prime}\right)} \check{G}\left(\mathbf{x}, \mathbf{x}^{\prime} ; i \omega_{m}\right) .
$$

Then, through an analytic continuation $i \omega_{m} \rightarrow \omega$, the Green's function becomes a function of complex frequency.

In the matrix structure adopted in Eq. (6), the $S P^{*} O T^{*}=$ -1 rule has the form:

$$
\hat{G}^{e h}\left(\mathbf{x}, \tau ; \mathbf{x}^{\prime}, \tau^{\prime}\right)=+\left[\hat{\sigma}_{y} \cdot \hat{G}^{e h}\left(\mathbf{x}^{\prime}, \tau^{\prime} ; \mathbf{x}, \tau\right) \cdot \hat{\sigma}_{y}\right]^{\top},
$$

or, equivalently, as a function of Matsubara frequency [63],

$$
\hat{G}^{e h}\left(\mathbf{x}, \mathbf{x}^{\prime} ; i \omega_{m}\right)=+\left[\hat{\sigma}_{y} \cdot \hat{G}^{e h}\left(\mathbf{x}^{\prime}, \mathbf{x} ;-i \omega_{m}\right) \cdot \hat{\sigma}_{y}\right]^{\top} .
$$

Therefore, Eq. (11b) implies for the singlet $\left[G^{e h}\right]^{s}$ and triplet components $\left[\mathbf{G}^{e h}\right]^{t}$ :

$$
\begin{aligned}
\hat{G}^{e h}\left(\mathbf{x}, \mathbf{x}^{\prime} ; i \omega_{m}\right)= & {\left[G^{e h}\right]^{s}\left(\mathbf{x}, \mathbf{x}^{\prime} ; i \omega_{m}\right) \hat{\sigma}_{0} } \\
& +\left(\left[\mathbf{G}^{e h}\right]^{t}\left(\mathbf{x}, \mathbf{x}^{\prime} ; i \omega_{m}\right) \cdot \hat{\vec{\sigma}}\right)
\end{aligned}
$$

the following symmetry properties under $P^{*}$ and $T^{*}$ :

$$
\begin{aligned}
{\left[G^{e h}\right]^{s}\left(\mathbf{x}, \mathbf{x}^{\prime} ; i \omega_{m}\right) } & =+\left[G^{e h}\right]^{s}\left(\mathbf{x}^{\prime}, \mathbf{x} ;-i \omega_{m}\right), \\
P^{*} T^{*} & =+1, \\
{\left[\mathbf{G}^{e h}\right]^{t}\left(\mathbf{x}, \mathbf{x}^{\prime} ; i \omega_{m}\right) } & =-\left[\mathbf{G}^{e h}\right]^{t}\left(\mathbf{x}^{\prime}, \mathbf{x} ;-i \omega_{m}\right), \\
P^{*} T^{*} & =-1 .
\end{aligned}
$$

This in accordance with the fact that spin-singlet pair correlations are odd under exchange of spin indices $(S=-1)$, while spin-triplet are even $(S=+1)$.

\section{Dyson equation}

The Green's function defined in Eq. (10) in the presence of impurity potential Eq. (2) satisfies the following Dyson equation:

$$
\begin{aligned}
\check{G}\left(\mathbf{x}, \mathbf{x}^{\prime} ; \omega\right)= & \check{G}_{\mathrm{cl}}\left(\mathbf{x}, \mathbf{x}^{\prime} ; \omega\right) \\
& +U_{m} \check{G}_{\mathrm{cl}}(\mathbf{x}, \mathbf{0} ; \omega) \cdot \check{P} \cdot \check{G}\left(\mathbf{0}, \mathbf{x}^{\prime} ; \omega\right),
\end{aligned}
$$

with

$$
\check{P}=\check{P}^{\dagger}=\check{P}^{-1}=\hat{\tau}_{0} \otimes(\mathbf{n} \cdot \hat{\vec{\sigma}}) .
$$

Here $\check{G}_{\mathrm{cl}}\left(\mathbf{x}, \mathbf{x}^{\prime} ; \omega\right)$ is the Green's function matrix for the clean system which is invariant under spatial translations:

$$
\check{G}_{\mathrm{cl}}\left(\mathbf{x}, \mathbf{x}^{\prime} ; \omega\right)=\frac{1}{L^{D}} \sum_{\mathbf{k}} \check{G}_{\mathrm{cl}}(\mathbf{k} ; \omega) e^{i \mathbf{k} \cdot\left(\mathbf{x}-\mathbf{x}^{\prime}\right)},
$$

and $\check{G}_{\mathrm{cl}}(\mathbf{k} ; \omega)$ is assumed to correspond to a conventional $s$-wave spin-singlet superconductor with a SC OP $\Delta$ and an isotropic normal dispersion $\xi_{k}$ invariant under spin rotations. This means that $\check{G}_{\mathrm{cl}}\left(\xi_{k} ; \omega\right)=\hat{G}_{\mathrm{cl}}\left(\xi_{k} ; \omega\right) \otimes \hat{\sigma}_{0}$, where:

$$
\begin{aligned}
\hat{G}_{\mathrm{cl}}^{-1}\left(\xi_{k} ; \omega\right) & =\omega \hat{\tau}_{0}-\xi_{k} \hat{\tau}_{3}-\hat{\Delta}, \\
\hat{\Delta} & =\Delta \hat{\tau}_{+}+\Delta^{*} \hat{\tau}_{-} .
\end{aligned}
$$

The integral over $\mathbf{k}$ in Eq. (14) is evaluated in the wide-band limit in Appendix A and the result is expressible in terms of the functions $\left(\phi_{c / s}\right)_{D}(r, \omega)$. Due to the translation invariance and isotropy, these functions only depend on $r=\left|\mathbf{x}-\mathbf{x}^{\prime}\right|$. The $\omega$ dependence enters through $\kappa(\omega)=\sqrt{|\Delta|^{2}-\omega^{2}} / v_{F}$, so their real part is even in $\omega$, while the imaginary part is odd. The real-space Green's function is given by:

$$
\begin{aligned}
- & \frac{1}{\pi N_{F}} \hat{G}_{\mathrm{cl}}\left(\mathbf{x}, \mathbf{x}^{\prime} ; \omega\right) \\
& =\left(\phi_{c}\right)_{D}(r ; \omega) \hat{g}_{0}(\omega)+\left(\phi_{s}\right)_{D}(r ; \omega) \hat{\tau}_{3}, \\
\hat{g}_{0}(\omega) & =\frac{\omega \hat{\tau}_{0}+\hat{\Delta}}{\sqrt{|\Delta|^{2}-\omega^{2}}},
\end{aligned}
$$

where $N_{F}$ is the DOS per spin component and its dependence on the model parameters is given by Eq. (A2). Note that $\left(\phi_{c}\right)_{D}(0 ; \omega)=1$ and $\left(\phi_{s}\right)_{D}(0 ; \omega)=0$.

Finally, the solution of Eq. (12) is of the form:

$$
\breve{G}\left(\mathbf{x}, \mathbf{x}^{\prime} ; \omega\right)=\breve{G}_{\mathrm{cl}}\left(\mathbf{x}, \mathbf{x}^{\prime} ; \omega\right)+\check{G}_{\mathrm{imp}}\left(\mathbf{x}, \mathbf{x}^{\prime} ; \omega\right),
$$

so that all the effects of the magnetic impurity potential are expressed in terms of the impurity Green's function $\check{G}_{\text {imp }}\left(\mathbf{x}, \mathbf{x}^{\prime} ; \omega\right)$. This function lacks translation symmetry due to the presence of a scattering center. Nevertheless, it has an 
analytic expression that may be obtained within the $T$-matrix approach. Details of its evaluation are given in Appendix B. The $T$-matrix has a denominator that is a quadratic function of $\omega$, giving two poles at $\pm \varepsilon_{0}$, corresponding to the energy of the YSR states. They are given by [27]:

$$
\varepsilon_{0}=\frac{1-J_{m}^{2}}{1+J_{m}^{2}}|\Delta|,
$$

with the dimensionless impurity strength:

$$
J_{m}=\pi N_{F} U_{m}
$$

\section{RESULTS}

In this section, we present results that are directly related to the pair correlations around the impurity and experimentally measurable single-particle properties.

\section{A. Analytic expression for the impurity Green's function}

We begin our analysis deriving the expression for the impurity Green's function using the $T$-matrix formalism, described Sec. B. From Eqs. (B1a), (B2), and (16a), we have the following explicit expression for the impurity correction to the Green's function matrix:

$$
\begin{aligned}
-\frac{1}{\pi N_{F}} \check{G}_{\mathrm{imp}}\left(\mathbf{x}, \mathbf{x}^{\prime} ; \omega\right)= & \frac{J_{m}}{D_{\mathrm{YSR}}(\omega)}\left[\hat{g}^{s}\left(\mathbf{x}, \mathbf{x}^{\prime} ; \omega\right) \otimes \hat{\sigma}_{0}\right. \\
& \left.+\hat{g}^{t}\left(\mathbf{x}, \mathbf{x}^{\prime} ; \omega\right) \otimes(\mathbf{n} \cdot \hat{\vec{\sigma}})\right],
\end{aligned}
$$

where

$$
D_{\mathrm{YSR}}(\omega)=\left(1+J_{m}^{2}\right)^{2} \omega^{2}-\left(1-J_{m}^{2}\right)^{2}|\Delta|^{2}
$$

is a denominator with poles at the YSR bound-state energies $\pm \varepsilon_{0}$, Eq. (18), and $\hat{g}^{S}\left(\mathbf{x}, \mathbf{x}^{\prime} ; \omega\right)$ and $\hat{g}^{t}\left(\mathbf{x}, \mathbf{x}^{\prime} ; \omega\right)$ denote the singlet and triplet component of the Green function. We note that the triplet component is aligned with the magnetization direction of the impurity. The singlet and triplet contributions can be decomposed in a basis of four functions with definite parity under the exchange $\mathbf{x} \leftrightarrow \mathbf{x}^{\prime}$ :

$$
\begin{aligned}
\psi_{1}\left(\mathbf{x}, \mathbf{x}^{\prime} ; \omega\right)= & \left(\phi_{c}\right)_{D}(r, \omega)\left(\phi_{c}\right)_{D}\left(r^{\prime}, \omega\right) \\
& +\left(\phi_{s}\right)_{D}(r, \omega)\left(\phi_{s}\right)_{D}\left(r^{\prime}, \omega\right), \\
\psi_{2}\left(\mathbf{x}, \mathbf{x}^{\prime} ; \omega\right)= & \left(\phi_{c}\right)_{D}(r, \omega)\left(\phi_{c}\right)_{D}\left(r^{\prime}, \omega\right) \\
& -\left(\phi_{s}\right)_{D}(r, \omega)\left(\phi_{s}\right)_{D}\left(r^{\prime}, \omega\right), \\
\psi_{3}\left(\mathbf{x}, \mathbf{x}^{\prime} ; \omega\right)= & \left(\phi_{s}\right)_{D}(r, \omega)\left(\phi_{c}\right)_{D}\left(r^{\prime}, \omega\right) \\
& +\left(\phi_{s}\right)_{D}\left(r^{\prime}, \omega\right)\left(\phi_{c}\right)_{D}(r, \omega), \\
\psi_{4}\left(\mathbf{x}, \mathbf{x}^{\prime} ; \omega\right)= & \left(\phi_{s}\right)_{D}(r, \omega)\left(\phi_{c}\right)_{D}\left(r^{\prime}, \omega\right) \\
& +\left(\phi_{s}\right)_{D}\left(r^{\prime}, \omega\right)\left(\phi_{c}\right)_{D}(r, \omega) .
\end{aligned}
$$

The first three functions are even, while the last is odd under coordinate exchange. All of the functions, according to Eqs. (A5), are even in frequency. Analytic expressions and the spatial behavior of these functions are investigated in more detail in Appendix $\mathrm{C}$ for different $D$ dimensions.
Using these basis functions, we may write $\hat{g}^{\alpha}\left(\mathbf{x}, \mathbf{x}^{\prime} ; \omega\right)=$ $\sum_{a=1}^{4} \hat{g}_{a}^{\alpha}(\omega) \psi_{a}\left(\mathbf{x}, \mathbf{x}^{\prime} ; \omega\right), \alpha \in(s, t)$, where:

$$
\begin{aligned}
& \frac{\hat{g}_{1}^{t}(\omega)}{1-J_{m}^{2}} \\
& =-\frac{\sqrt{|\Delta|^{2}-\omega^{2}} \hat{g}_{1}^{s}(\omega)}{2 J_{m} \omega} \\
& =|\Delta|^{2} \hat{\tau}_{0}+\omega \hat{\Delta}, \\
& \frac{\hat{g}_{2}^{t}(\omega)}{\left(1+J_{m}^{2}\right) \omega \sqrt{|\Delta|^{2}-\omega^{2}}} \\
& =-\frac{\hat{g}_{2}^{s}(\omega)}{J_{m}\left[\left(1+J_{m}^{2}\right) \omega^{2}+\left(1-J_{m}^{2}\right)|\Delta|^{2}\right]} \\
& =\hat{g}_{0}(\omega), \\
& \frac{\left(1+J_{m}^{2}\right) \omega \sqrt{|\Delta|^{2}-\omega^{2}}}{\hat{g}_{3}^{t}(\omega)} \\
& =-\frac{g_{3}^{s}(\omega)}{J_{m}\left[\left(1+J_{m}^{2}\right) \omega^{2}+\left(1-J_{m}^{2}\right)|\Delta|^{2}\right]} \\
& =\hat{\tau}_{3}, \\
& \frac{\hat{g}_{4}^{t}(\omega)}{\left(1-J_{m}^{2}\right) \sqrt{|\Delta|^{2}-\omega^{2}}} \\
& =-\frac{\hat{g}_{4}^{s}(\omega)}{2 J_{m} \omega}=\hat{\tau}_{3} \hat{\Delta} . \\
& =
\end{aligned}
$$

\section{B. Reciprocal relations between singlet and triplet pair correlations}

The $S P^{*} O T^{*}=-1$ condition for single-band systems, where $O=+1$, goes over to $S P^{*} T^{*}=-1$. Thus, there are four possibilities of allowed combined symmetries $\left(S, P^{*}, T^{*}\right)$. The enumeration of these possibilities, together with their labeling and the corresponding contributions from Eqs. (16) and (23) are given in Table I. We use the subindex $\left(S, P^{*}, T^{*}\right)$, with $S, P^{*}, T^{*}= \pm$, to denote the part of the anomalous Green's function (eh component) with even/odd symmetry under the exchange of the corresponding labels. The components $\left[g_{a}^{t}\right]^{e h}(\omega)$ for $a=1,2$ ( $a=3$ does not have an electron-hole components) are necessarily odd in $\omega$, while $\left[g_{4}^{t}\right]^{e h}(\omega)$ is even in $\omega$. For $\left[g_{a}^{s}\right]^{e h}(\omega)$, the situation is reversed. Having in mind the general matrix structure of $\hat{g}_{a}^{t}$ and $\hat{g}_{a}^{s}$ given in Eqs. (23), we see that for any pair of coordinates $\mathbf{x}, \mathbf{x}^{\prime}$, there is a general reciprocity relation between the singlet and

TABLE I. The allowed symmetries under $\left(S, P^{*}, T^{*}\right)$ for the electron-hole propagators $\hat{G}^{e h}\left(\mathbf{x}, \mathbf{x}^{\prime} ; \omega\right)$ and the corresponding contributions. The different symmetries under $P^{*}$ are split, indicating the reciprocity in exchange of the symmetry under $S$ and $T^{*}$.

\begin{tabular}{ccccc}
\hline \hline$S$ & $P^{*}$ & $T^{*}$ & Notation & Contribution \\
\hline- & + & + & $G_{(-,+,+)}^{e h}\left(\mathbf{x}, \mathbf{x}^{\prime} ; \omega\right)$ & $\hat{g}_{0}(\omega), \hat{g}_{1}^{s}(\omega), \hat{g}_{2}^{s}(\omega)$ \\
+ & + & - & $G_{(+,+,-)}^{\text {hh }}\left(\mathbf{x}, \mathbf{x}^{\prime} ; \omega\right)$ & $\hat{g}_{1}^{t}(\omega), \hat{g}_{2}^{t}(\omega)$ \\
+ & - & + & $G_{(+,-,+}^{e h}\left(\mathbf{x}, \mathbf{x}^{\prime} ; \omega\right)$ & $\hat{g}_{4}^{t}(\omega)$ \\
- & - & - & $G_{(-,-,-)}^{\text {eh }}\left(\mathbf{x}, \mathbf{x}^{\prime} ; \omega\right)$ & $\hat{g}_{4}^{s}(\omega)$ \\
\hline \hline
\end{tabular}


triplet components with different parity in frequency $\omega$. We point out, however, that the basis functions Eqs. (22) also carry (even) frequency dependence, so the total frequency dependence is coordinate dependent.

Another reciprocity between the electron-hole components follows by noticing that the same ratio holds:

$$
\begin{aligned}
& \hat{g}_{a}^{s}(\omega)=r_{I}(\omega) \hat{g}_{a}^{t}(\omega),(a=1,4), \\
& r_{I}(\omega)=-\frac{2 J_{m} \omega}{\left(1-J_{m}^{2}\right) \sqrt{|\Delta|^{2}-\omega^{2}}},
\end{aligned}
$$

for two sets of basis functions with opposite parity under coordinate exchange. We note that at $\omega \rightarrow 0$ the ratio vanishes, implying that $\hat{g}_{a}^{s}(\omega=0)=0(a=1,4)$. In the same way, at the critical impurity strength, $J_{m}=1, \hat{g}_{a}^{t}(\omega)=0(a=1,4)$. Taking the electron-hole components, we may rewrite the ratio in Eq. (24a) as:

$$
\frac{\left[g_{4}^{s}\right]^{e h}(\omega)}{\left[g_{1}^{s}\right]^{e h}(\omega)}=\frac{\left[g_{4}^{t}\right]^{e h}(\omega)}{\left[g_{1}^{t}\right]^{e h}(\omega)}=\frac{\sqrt{|\Delta|^{2}-\omega^{2}}}{\omega} .
$$

Thus, there is a reciprocity between exchange of the parity with respect to coordinate exchange ( $a=1$ and $a=4$ ) and $\omega$, while keeping the same spin symmetry. The ratio of these pair correlations is independent of the impurity strength $J_{m}$.

A similar relation can be derived for the remaining two basis functions with same parity under coordinate exchange

$$
\begin{aligned}
& \hat{g}_{a}^{s}(\omega)=r_{\mathrm{II}}(\omega) \hat{g}_{a}^{t}(\omega),(a=2,3), \\
& r_{\mathrm{II}}(\omega)=-\frac{J_{m}\left[\left(1+J_{m}^{2}\right) \omega^{2}+\left(1-J_{m}^{2}\right)|\Delta|^{2}\right]}{\left(1+J_{m}^{2}\right) \omega \sqrt{|\Delta|^{2}-\omega^{2}}} .
\end{aligned}
$$

We note that $\left[g_{3}^{\alpha}\right]^{e h}(\omega)=0$, which may be interpreted as a selection rule, which guarantees that there is no electron-hole component generated by impurity scattering if neither the spin nor the parity symmetry is changed.

\section{Spin-polarized local density of states}

We begin plotting local single-particle properties, such as the SP LDOS. The LDOS $v(\mathbf{x}, \omega)$ and the LMDOS $\mathbf{m}(\mathbf{x}, \omega)$ are evaluated as:

$$
\begin{aligned}
& v(\mathbf{x}, \omega) \\
& \quad=-\frac{1}{\pi} \lim _{\eta \rightarrow 0} \operatorname{Im}\left[\operatorname{Tr}\left\{\hat{G}^{e e}(\mathbf{x}, \mathbf{x}, \omega+i \eta)\right\}\right], \\
& \mathbf{m}(\mathbf{x}, \omega) \\
& \quad=-\frac{1}{\pi} \lim _{\eta \rightarrow 0} \operatorname{Im}\left[\operatorname{Tr}\left\{\hat{\vec{\sigma}} \cdot \hat{G}^{e e}(\mathbf{x}, \mathbf{x}, \omega+i \eta)\right\}\right] .
\end{aligned}
$$

The magnetization has the same direction as the impurity spin polarization. The spin-up $(\sigma=+1)$ and spin-down $(\sigma=$ $-1)$ components of the SP LDOS are evaluated as $v_{\sigma}=(v+$ $\sigma|\mathbf{m}|) / 2$ and can be experimentally measured with a magnetic tip STM in spectroscopic mode. From them, working backwards, one may determine $\mathbf{m}$.

When representing intensity plots of a quantity $f$ (SP LDOS, LMDOS, or real or imaginary part of a particular pair correlation) containing narrow high-intensity peaks around the YSR bound-state energies or the gap edges, and having
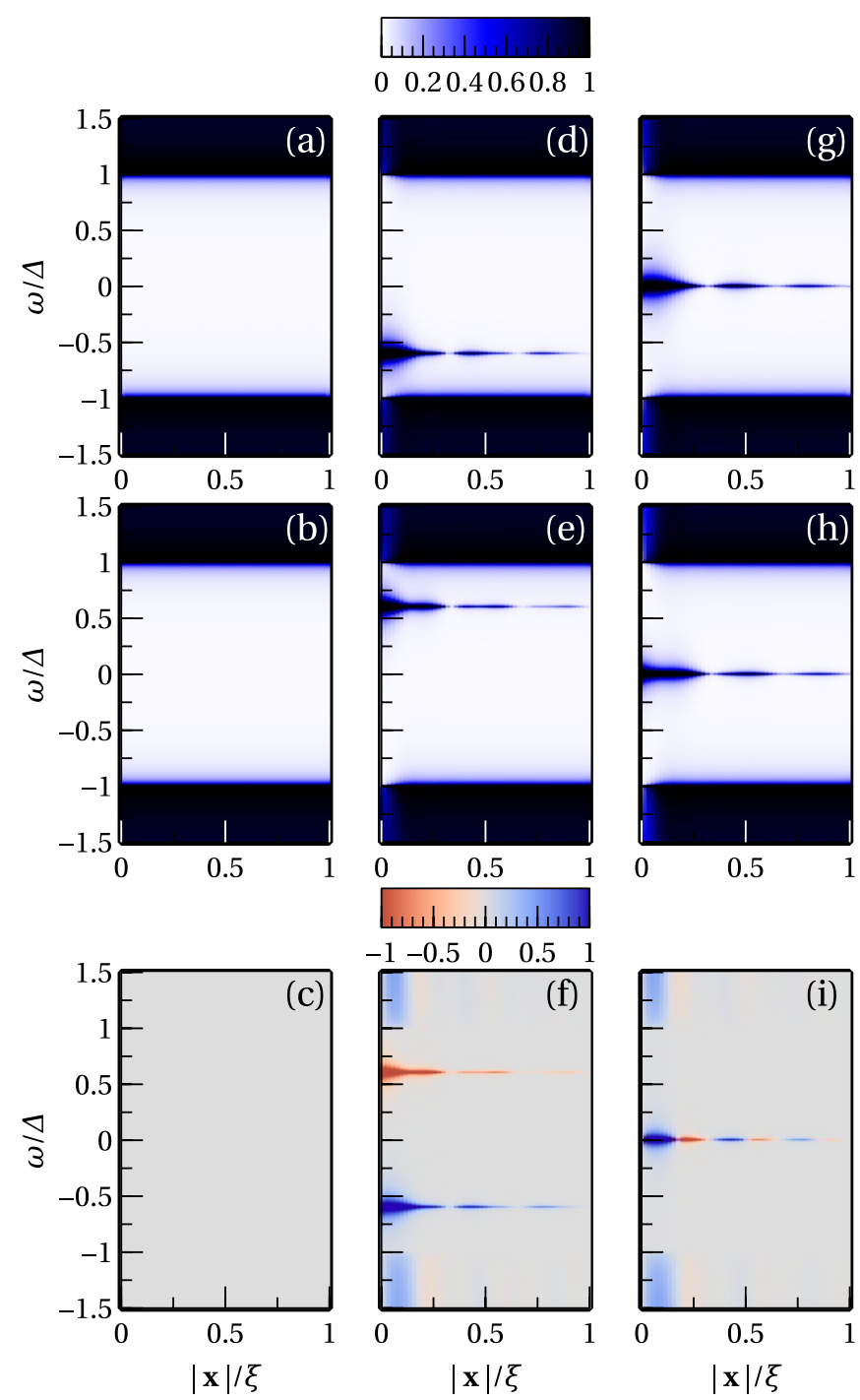

FIG. 2. The spin-up LDOS [(a), (d), and (g)], spin-down LDOS $[(\mathrm{b}),(\mathrm{e})$, and (h)], and the LMDOS [(c), (f), and (i)] for a range of values of $|\mathbf{x}| / \xi$ in $D=3$, and $\omega / \Delta$. Each column corresponds to a different value of the dimensionless impurity strength parameter, $J_{m}=0$ [(a), (b), and (c)], $J_{m}=0.5\left[(\mathrm{~d}),(\mathrm{e})\right.$, and (f)], and $J_{m}=1.0$ $\left[(\mathrm{g}),(\mathrm{h})\right.$, and (i)]. The SC coherence length is $\xi / \lambda_{F}=3$, and $f_{0}$ in Eq. (27) is $f_{0}=0.27$. The gray color coding of panel (c) indicates no magnetization.

a smooth background, we use a scaling factor $s$ according to the sigmoid scaling function:

$$
s(f)=\operatorname{sgn}(f)\left[1-\exp \left(-\frac{|f|}{f_{0}}\right)\right] .
$$

This is an odd-valued $[s(-f)=-s(f)]$, smooth singleparameter function, where the parameter $f_{0}$ roughly corresponds to a region of values for $f$ for which the scaling is linear. This scaling has the advantage that it collapses the whole real line on a finite segment $[-1,1]$, while keeping a linear resolution for low magnitudes, which is particularly convenient in plots with narrow isolated peaks and a varying background. 
In Fig. 2, we display the spin-up LDOS [Figs. 2(a), 2(d), and 2(g)], the spin-down LDOS [Figs. 2(b), 2(e), and 2(h)], and the LMDOS [Figs. 2(c), 2(f), and 2(i)] as function of the frequency $\omega / \Delta$ and the position $|\mathbf{x}| / \xi$ away from the impurity.

For illustration purposes, we take a short SC coherence length $\xi / \lambda_{F}=3$, which implies a value for the SC OP in units of the Fermi energy $\left(\varepsilon_{F}\right), \Delta / \varepsilon_{F}=\lambda_{F} /(2 \pi \xi)=0.053$. Also, we evaluate the Green's function at $\omega \rightarrow \omega+i \eta$, where $\eta / \Delta=0.0005$ in Eqs. (26) to avoid divergencies. Each column in Fig. 2 corresponds to a different value of the dimensionless impurity strength $J_{m}$. The energy $\varepsilon_{0}$ of the YSR bound state, Eq. (18), changes sign when $J_{m} \rightarrow 1 / J_{m}$. This has the effect of switching the subgap peaks in any of the Figs. 2-8, including their sign, as was explicitly verified by comparing the middle column panels with analogous plots for $J_{m}=2.0$.

For a clean system, $J_{m}=0$ (left column), there is no spin polarization and the LDOS is homogeneous with BCS coherence peaks at $\pm|\Delta|$. As the impurity strength is increased (middle column), two subgap SP peaks appear at $\pm \varepsilon_{0}$ : YSR bound states. Below the critical impurity strength $\left(J_{m}<1\right)$, the state at $-\varepsilon_{0}$ has the same spin polarization as the impurity, while the one at $\varepsilon_{0}$ has the opposite polarization. The spin polarization of these peaks does not change direction with position. At the same time, the above-gap continuum shows a small spin polarization that has the same direction as the impurity for both positive and negative frequencies but oscillates with position according to the oscillations of the basis functions (Fig. 9).

At critical impurity strength, $J_{m}=1$ (right column), the two subgap peaks merge at zero frequency. Remarkably, they do not cancel but instead display oscillation in the spin polarization with position. As it will be shown in the following, similar features are also observed in the spin-triplet odd- $\omega$ local pair correlation. Finally, for $J_{m}>1$, the YSR states cross and their polarization is reversed.

\section{Local pair correlations spectra}

Guided by the pair correlations of the conventional SC OP, which are on-site, we first consider local $\left(\mathbf{x}=\mathbf{x}^{\prime}\right)$ pair correlations in the vicinity of the impurity. For this choice, the symmetry under exchange of coordinates is necessarily even $P^{*}=+1$, so there are only two possible components consistent with the $S P^{*} T^{*}=-1$ rule, namely even- $\omega$ spin-singlet $G_{(-,+,+)}^{e h}(\mathbf{x}, \mathbf{x}, \omega)$ and odd- $\omega$ spin-triplet $G_{(+,+,-)}^{e h}(\mathbf{x}, \mathbf{x}, \omega)$.

The conventional correlation belongs to the contribution $G_{(-,+,+)}^{e h}$, displayed in Fig. 3. In the clean limit [(a) and (b)], the system is uniform and there is no $\mathbf{x}$ dependence. The correlation is real and even- $\omega$ for subgap frequencies $(-|\Delta|<$ $\omega<|\Delta|$ ), while it becomes imaginary and odd for above-gap frequencies, as expected from a conventional superconductor. As the impurity strength is increased from $J_{m}=0[(\mathrm{c})$, (d), (e), and (f)], the features above the bulk gap persist. At intermediate impurity strengths, the build-up of YSR states at $\pm \varepsilon_{0}$ [for $J_{m}=0.5 \varepsilon_{0}=0.6|\Delta|$ in Figs. 3(c) and 3(d)] leads to additional features inside the superconducting gap. The real part of the $(-,+,+)$ component changes sign on both sides of the peak while the imaginary part has the same sign. This feature persists for the real and the imaginary part of any correlation function around the YSR, Figs. 4-8. Further, we
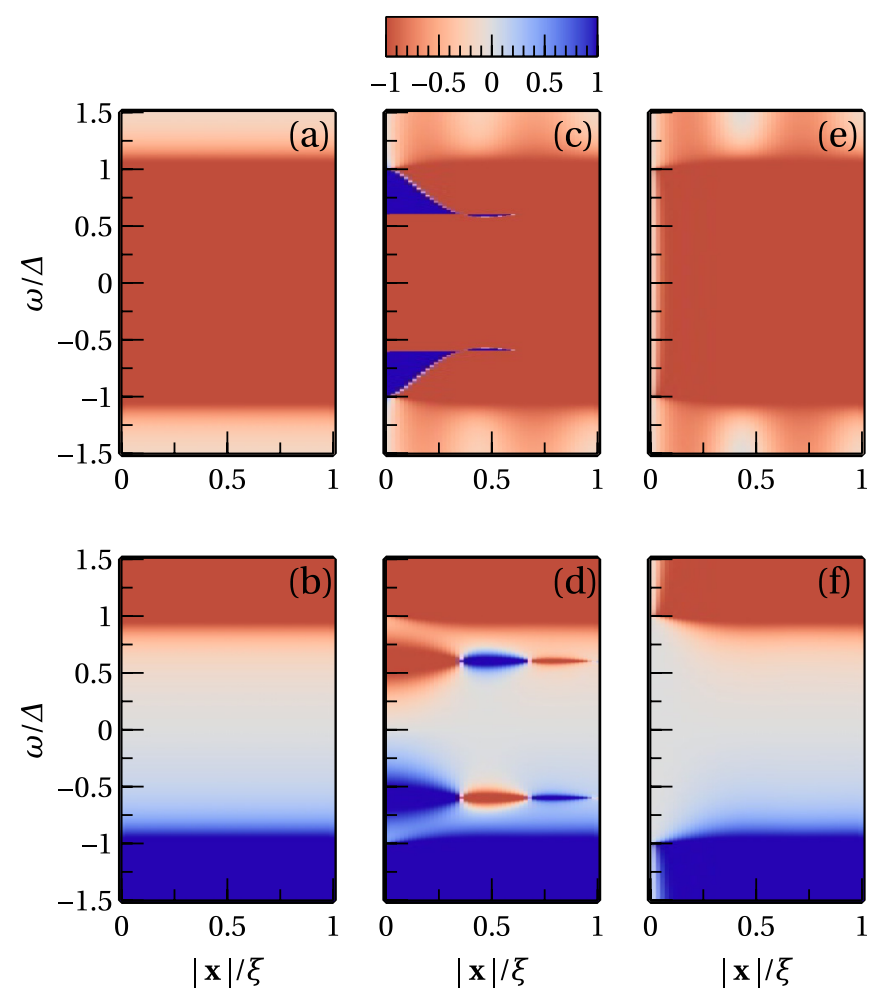

FIG. 3. Density plots for the real [(a), (c), and (e)] and imaginary parts [(b), (d), and (f)] of $-\frac{1}{\pi N_{F}} G_{(-,+,+)}^{e h}(\mathbf{x}, \mathbf{x}, \omega+i \eta)$ for a range of values of $|\mathbf{x}| / \xi$ and $\omega / \Delta$. The choice of parameters is the same as in Fig. 2, and $f_{0}$ in Eq. (27) is $f_{0}=0.60$.

note that the parity of the real and imaginary parts of any correlation are opposite [63]. The real part, being even in frequency, has the same sign between the YSR bound-state energies $\left(|\omega|<\varepsilon_{0}\right)$ and changes sign as the frequency crosses the YSR bound-state energies, vanishing at the gap edges $\left(\varepsilon_{0}<|\omega|<\Delta\right)$. The imaginary part, being odd in frequency, has sharp peaks around the YSR bound-state energies with opposite sign and spatial modulation determined by that of Fig. 9. As the strength of the impurity approaches the critical value, $J_{m}=1$, the two peaks with opposite sign merge at zero energy and cancel out. Therefore, the conventional superconducting correlations, $(-,+,+)$, are suppressed for the subgap frequency region and in the proximity to the impurity. For $J_{m}>1$ the subgap features appear again, showing the same symmetry as in the $J_{m}=0.5$ case, with a global sign in the imaginary part.

The odd- $\omega$ on-site component $G_{(+,+,-)}^{e h}$ is displayed in Fig. 4. Here we point out only the differences with Fig. 3. We note that the correlation is induced by the effect of the impurity (only from $\left[G_{\text {imp }}^{t}\right]^{\text {eh }}$ ) and, therefore, completely vanishes for a clean superconductor $\left(J_{m}=0\right)$. At finite $J_{m}$, we observe the appearance of signatures outside and inside the gap. This component is triplet with spin polarization in the direction of the impurity, as illustrated by Eq. (20). As shown in Fig. 4, the real part is odd (and the imaginary part is even) in $\omega$. Differently from the previously commented case, as the peaks merge [Fig. 4(f)], they do not cancel but enhance instead. The same enhancement persists also in the real part. The spatial modulation is identical to the one of Fig. 3, 

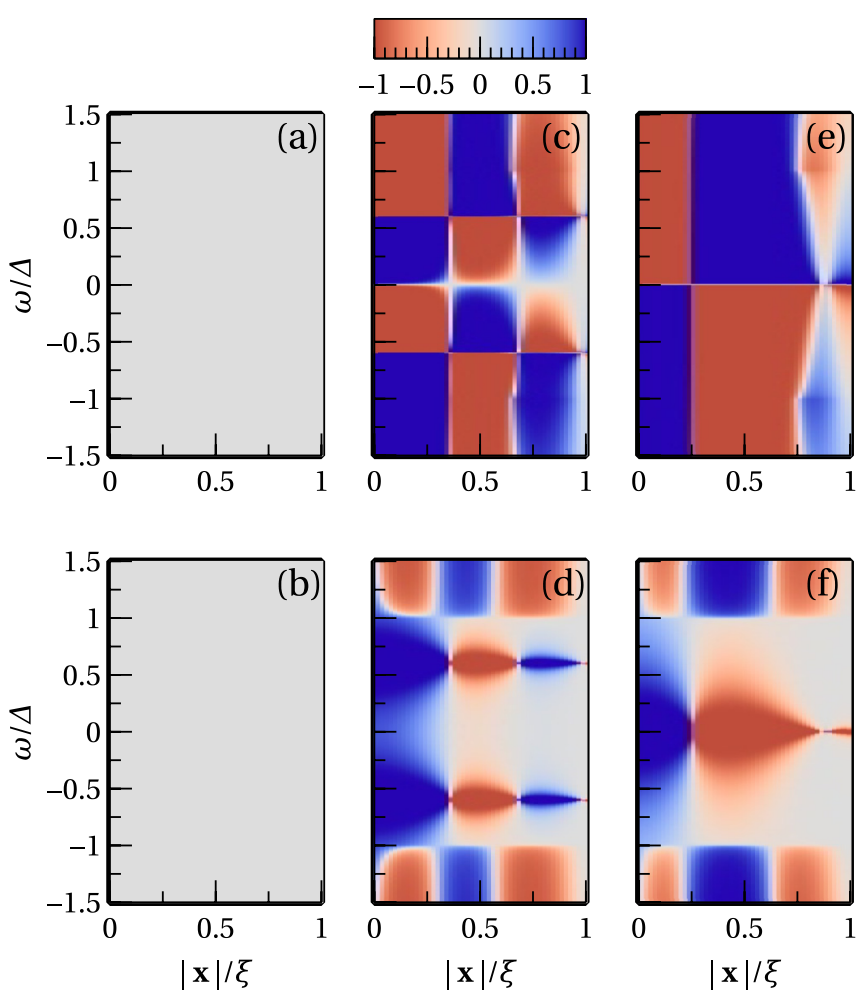

FIG. 4. Density plots for the real [(a), (c), and (e)] and imaginary parts [(b), (d), and (f)] of $-\frac{1}{\pi N_{F}} G_{(+,+,-)}^{e h}(\mathbf{x}, \mathbf{x}, \omega+i \eta)$ for a range of values of $|\mathbf{x}| / \xi$ and $\omega / \Delta$. The choice of parameters is the same as in Fig. 2, and $f_{0}$ in Eq. (27) is $f_{0}=0.11$. The gray color coding of the panels in the left column [(a) and (b)] indicates a zero value for the given value of the impurity strength.

which is in agreement with the reciprocity of the two matrices having the same spatial dependence. One final feature is the small contribution of the continuum states exhibiting the same spatial modulation as the YSR.

It is important to note that at the critical impurity strength the conventional $(-,+,+)$ pair correlation is completely suppressed in the vicinity of the impurity, as shown in Figs. 3(e) and 3(f). Therefore the only local component that survives is the $(+,+,-)$, which corresponds to the Berezinskii pairing [Figs. 4(e) and 4(f)].

\section{E. Nonlocal pair correlations spectra}

Another possibility to consider is the nonlocal pair correlations. Because the impurity site is preferential, we are interested in fixing one of the coordinates at the impurity site $\mathbf{x}^{\prime}=\mathbf{0}$. In Figs. 5-8, we show the electron-hole separation, when a hole is created at the impurity site and an electron is annihilated at a site $\mathbf{x}$.

Figure 5 displays the pair correlation with the same symmetry as the one in Fig. 3. In the absence of an impurity [Figs. 5(a) and 5(b)], it displays a characteristic periodicity with a period $\xi$. The spatial modulation is according to the different behavior of the basis functions for this choice of coordinates (Fig. 10). Unlike the local correlations, where the imaginary part is zero for subgap frequencies, in this case there is a finite component that decays with separation
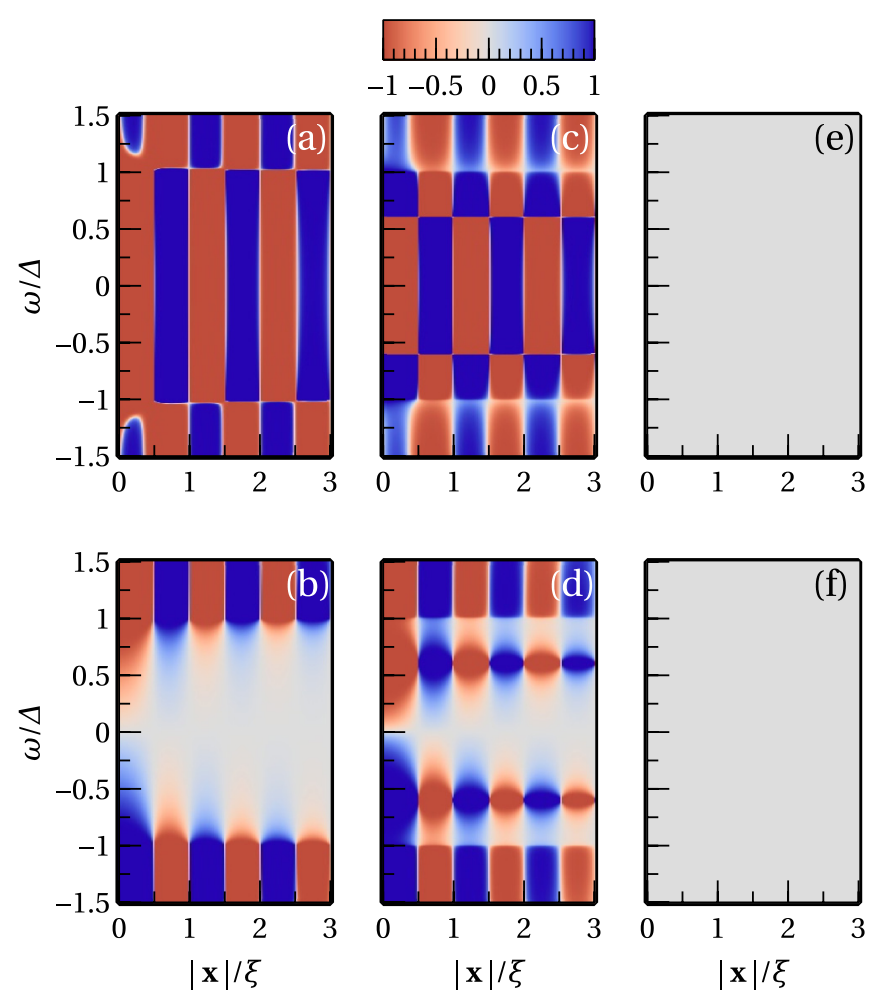

FIG. 5. Density plots for the real [(a), (c), and (e)] and imaginary parts [(b), (d), and (f)] of $-\frac{1}{\pi N_{F}} G_{(-,+,+)}^{e h}(\mathbf{x}, \mathbf{0}, \omega+i \eta)$ for a range of values of $|\mathbf{x}| / \xi$ and $\omega / \Delta$. The choice of parameters is the same as in Fig. 2, and $f_{0}$ in Eq. (27) is $f_{0}=0.071$.

over a length scale determined by $1 / \operatorname{Re}[\kappa(\omega)]$, introduced in relation to Eqs. (16). As expected, the frequency dependence, including the parity and the behavior around the YSR boundstate energies is the same as in the local case, since it is determined by the basis functions. Thus, at critical impurity strength, $J_{m}=1$ [Fig. 5(e) and 5(f)], the imaginary and real parts vanish in accordance with the exact cancellation of the local correlation at the impurity site.

An analogous comparison may be made for the nonlocal (Fig. 6) and local (Fig. 4) correlations with symmetry $(+,+,-)$. It exhibits the same spatial dependence as in Fig. 5. As in Fig. 4, the real part of the correlation is odd in frequency while the imaginary one is even. Similarly to the local case, the $(+,+,-)$ correlation does not vanish for $J_{m}=1$ but increases instead. It means that, close to the critical impurity strength, the odd correlations dominate.

In addition to the two components discussed before, there are two more correlation components appearing in the nonlocal case. According to Table I, they correspond to the case where the correlation is odd under exchange of spatial coordinates $\left(P^{*}=-1\right)$. They are displayed in Figs. 7 and 8, respectively.

As shown, both of the odd-parity nonlocal pair correlations require a nonzero impurity scattering, as illustrated by the vanishing of Figs. 7(a) and 7(b) and Figs. 8(a) and 8(b). For $J_{m} \neq 1$, both components exhibit a finite value, where the symmetry of the real part is set by the $T^{*}$ value. For $J_{m}=1$, the $(+,-,+)$ component vanishes while the $(-,-,-)$ odd- $\omega$ gets enhanced. By comparing the behavior at critical 

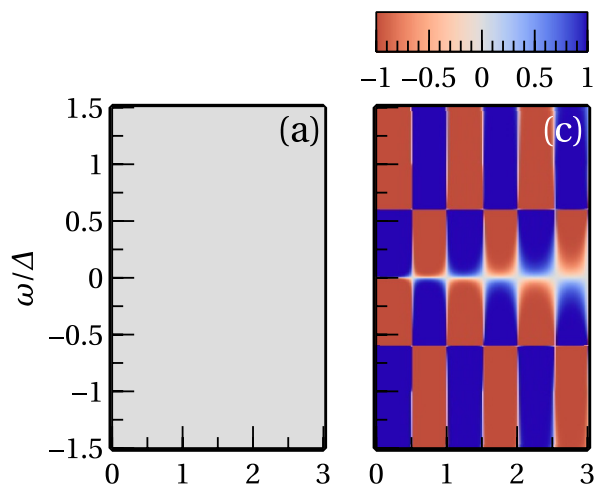

$$
\begin{array}{lllll}
-1 & -0.5 & 0 & 0.5 & 1
\end{array}
$$
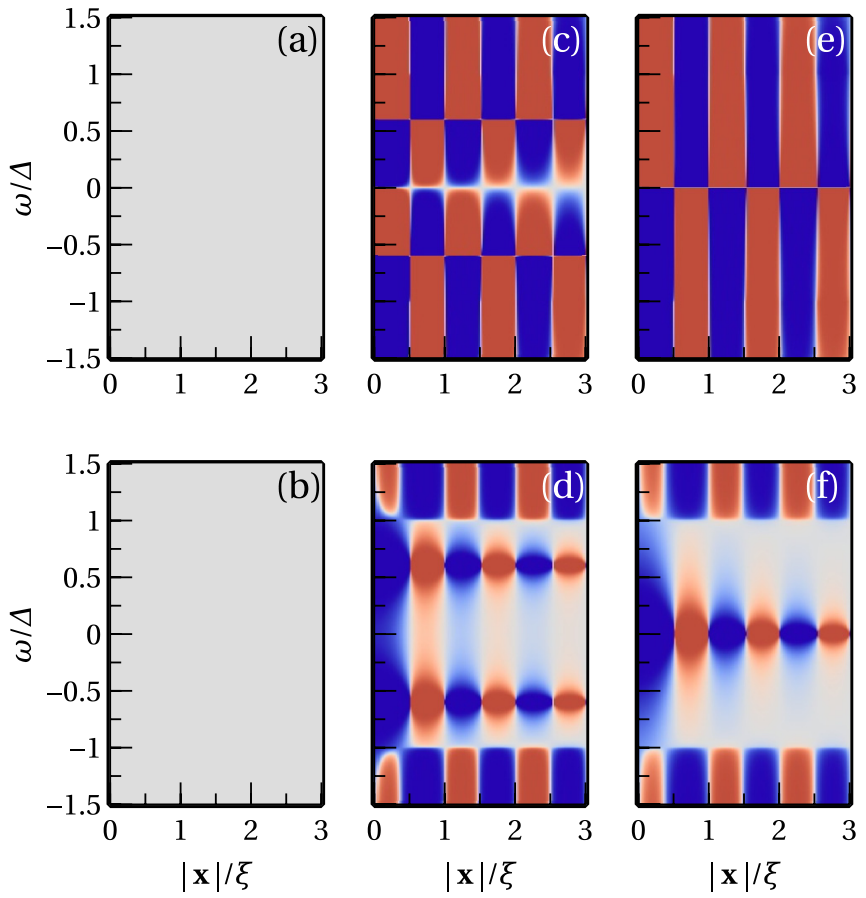

FIG. 6. Density plots for the real [(a), (c), and (e)] and imaginary parts $\left[(\mathrm{b}),(\mathrm{d})\right.$, and (f)] of $-\frac{1}{\pi N_{F}} G_{(+,+,-)}^{e h}(\mathbf{x}, \mathbf{0}, \omega+i \eta)$ for a range of values of $|\mathbf{x}| / \xi$ and $\omega / \Delta$. The choice of parameters is the same as in Fig. 2, and $f_{0}$ in Eq. (27) is $f_{0}=0.064$.
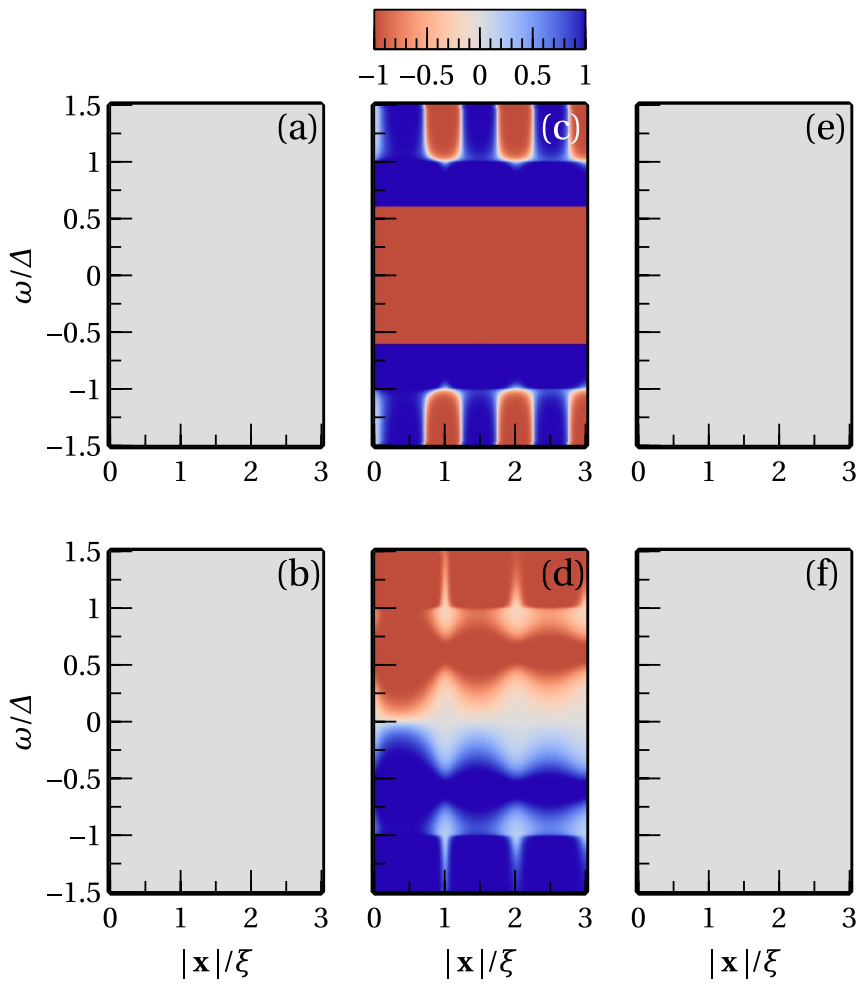

FIG. 7. Density plots for the real [(a), (c), and (e)] and imaginary parts $\left[(\mathrm{b}),(\mathrm{d})\right.$, and (f)] of $-\frac{1}{\pi N_{F}} G_{(+,-,+)}^{e h}(\mathbf{x}, \mathbf{0}, \omega+i \eta)$ for a range of values of $|\mathbf{x}| / \xi$ and $\omega / \Delta$. The choice of parameters is the same as in Fig. 2, and $f_{0}$ in Eq. (27) is $f_{0}=0.020$.
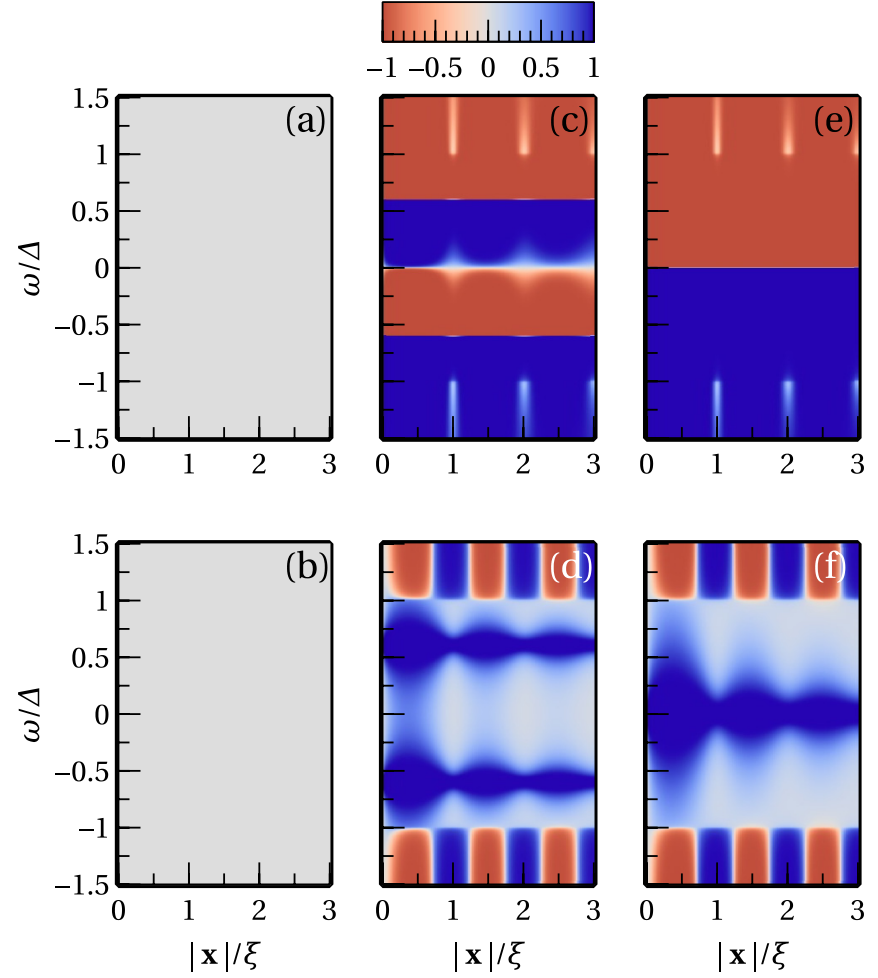

FIG. 8. Density plots for the real [(a), (c), and (e)] and imaginary parts [(b), (d), and (f)] of $-\frac{1}{\pi N_{F}} G_{(-,-,-)}^{e h}(\mathbf{x}, \mathbf{0}, \omega+i \eta)$ for a range of values of $|\mathbf{x}| / \xi$ and $\omega / \Delta$. The choice of parameters is the same as in Fig. 2, and $f_{0}$ in Eq. (27) is $f_{0}=0.062$.

impurity strength on Figs. 7(e) and 7(f), and Figs. 5(e) and 5(f), we conclude that there exists another type of reciprocity. In this case the parity and spin symmetry are exchanged, while keeping the frequency symmetry unchanged, as noted in Sec. III B. We note that the odd-parity pair correlations have spatial dependence determined by the odd basis function $\psi_{4}$, which has a different spatial behavior than the even ones (Fig. 10), with no change in sign in both the real and imaginary parts for subgap frequencies.

\section{CONCLUSIONS}

To conclude, we have demonstrated that the YSR bound states formed at the vicinity of magnetic impurities in conventional superconductors harbor spin-triplet, odd- $\omega$ pair correlations, which may be referred to as Berezinskii-YSR (BYSR) states. In case of spin-rotation-invariant bulk SC, the spin polarization of the BYSR state is collinear with the impurity spin. We have demonstrated that the odd-frequency pairing dominates close to the impurity at the critical coupling strength, where YSR states merge at the middle of the gap. This leads to a regime where odd-frequency correlations can be probed experimentally. We note that the local oddfrequency component exhibits the same frequency and spatial dependence than the local magnetization density of states, which can be measured using spin-polarized scanning tunneling spectroscopy. By exactly solving the impurity Green's function, we were able to identify a complete reciprocity, independent from the impurity coupling strength, under 



FIG. 9. Real (solid lines) and imaginary (dashed lines) part of the retarded $(\omega \rightarrow \omega+i \eta)$ basis functions $\psi_{a}$ Eqs. (22) as a function of $|\mathbf{x}| / \xi$ for $\mathbf{x}=\mathbf{x}^{\prime}$ and $D=3$ dimensions. We represent $\psi_{1}$ (black lines), $\psi_{2}$ (red lines), and $\psi_{3}$ (green lines). We note $\psi_{4}=0$ as it is odd under position exchange. The SC OP is chosen so that $\xi / \lambda_{F}=3$. Different panels are fixed at different frequency: Panel $\omega / \Delta=0$ (a), $\omega / \Delta=0.5$ (b), $\omega / \Delta=1.0(\mathrm{c})$, and $\omega / \Delta=2.0(\mathrm{~d})$.

exchange of the $S$ and $T^{*}$ symmetries and with the same parity coordinate, $P^{*}$. Finally, we have also demonstrated the presence of all the allowed nonlocal pair correlations consistent with the $S P^{*} O T^{*}=-1$ rule and summarized in
Table I, showing that even-parity correlations are suppressed at the critical impurity strength.

Note added. After the submission of this paper we learned about the work of V. Perrin et al. [64], where similar
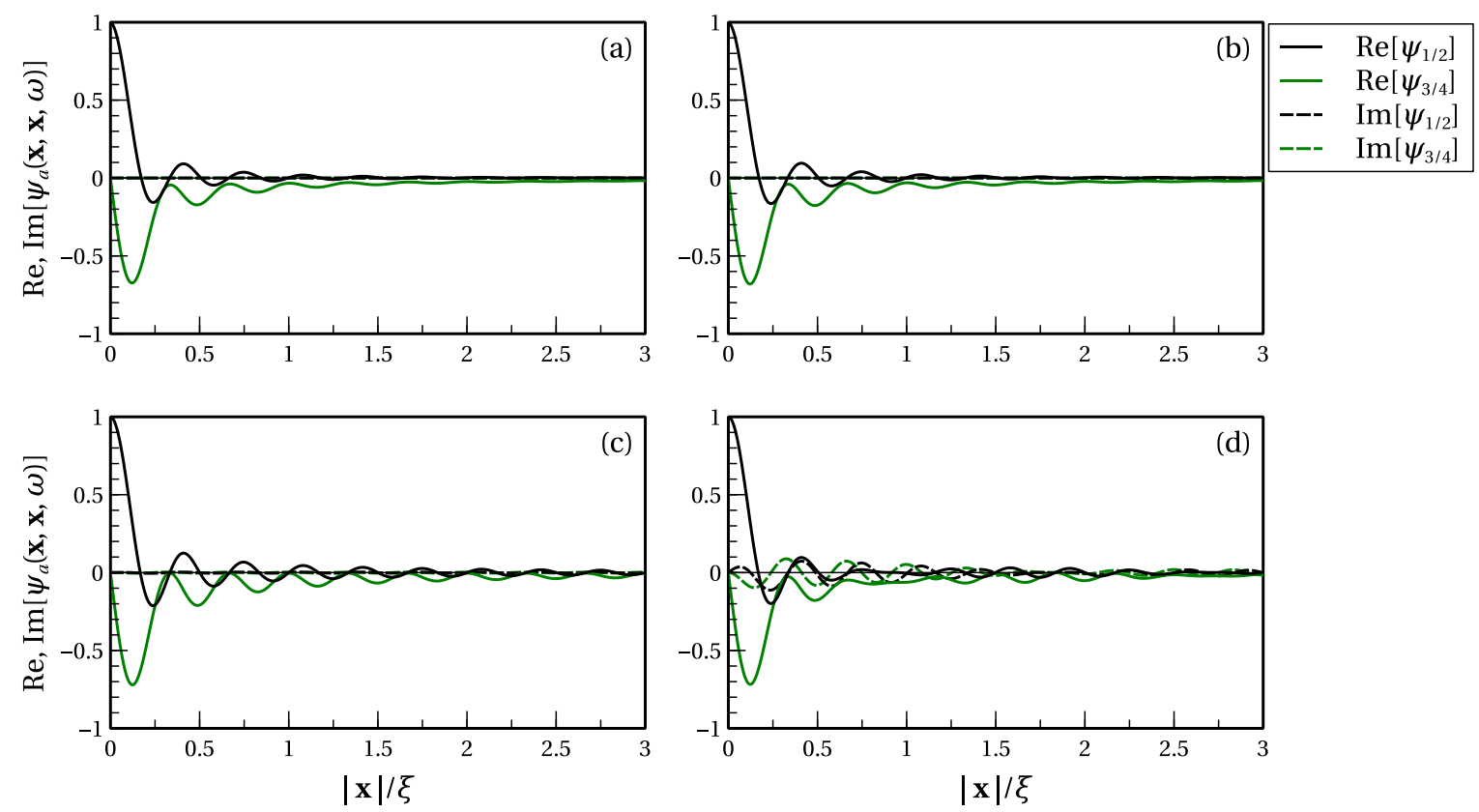

FIG. 10. Real (solid lines) and imaginary (dashed lines) part of the retarded $(\omega \rightarrow \omega+i \eta)$ basis functions $\psi_{a}$ Eqs. (22) as a function of $|\mathbf{x}| / \xi$ for $\mathbf{x}^{\prime}=\mathbf{0}$ and $D=3$ dimensions. The black line represents the behavior for $a=1$ and $a=2$ while the green one represents the $a=3$ and $a=4$ functions. The SC OP is chosen so that $\xi / \lambda_{F}=3$. Different panels are fixed at different frequencies: $\omega / \Delta=0$ (a), $\omega / \Delta=0.5$ (b), $\omega / \Delta=1.0(\mathrm{c})$, and $\omega / \Delta=2.0(\mathrm{~d})$. 
conclusions were reached about the existence of odd- $\omega$ pairing near a magnetic impurity site.

\section{ACKNOWLEDGMENTS}

The authors thank A. Khajetoorians and J. Mentink for useful discussions. D.K. and A.V.B. acknowledge funding by VILLUM FONDEN via the Centre of Excellence for Dirac Materials (Grant No. 11744), Knut and Alice Wallenberg Foundation (Grant No. KAW 2018.0104), and the European Research Council ERC-2018-SyG HERO. R.S.S. acknowledges funding from QuantERA project 2D hybrid materials as a platform for topological quantum computing.

\section{APPENDIX A: REAL SPACE CLEAN SUPERCONDUCTOR GREEN'S FUNCTION IN THE WIDE-BAND APPROXIMATION}

The real space Green's function is obtained by inverting Eq. (15a) and performing the momentum integral according to Eq. (14). When performing the momentum integral the following steps are taken:

$$
\begin{aligned}
\frac{1}{L^{D}} & \sum_{\mathbf{k}} e^{i k r \cos \theta} \hat{G}_{\mathrm{cl}}\left(\xi_{k} ; \omega\right) \\
= & \frac{\Omega_{D}}{(2 \pi)^{D}} \int \frac{d \Omega_{\hat{\mathbf{k}}}}{\Omega_{D}} \int_{0}^{\infty} d k k^{D-1} e^{i k r \cos \theta} \hat{G}_{\mathrm{cl}}\left(\xi_{k} ; \omega\right) \\
\approx & \frac{\Omega_{D} k_{F}^{D-1}}{(2 \pi)^{D} v_{F}} \int \frac{d \Omega_{\hat{\mathbf{k}}}}{\Omega_{D}} e^{i k_{F} r \cos \theta} \\
& \times \int_{-k_{F} v_{F}}^{\infty} d \xi\left(1+\frac{\xi}{k_{F} v_{F}}\right)^{D-1} e^{i \frac{r \cos \theta}{v_{F}} \xi} \hat{G}_{\mathrm{cl}}(\xi ; \omega) \\
\approx & N_{F} \int \frac{d \Omega_{\hat{\mathbf{k}}}}{\Omega_{D}} e^{i k_{F} r \cos \theta} \int_{-\infty}^{\infty} d \xi e^{i \frac{r \cos \theta}{v_{F}} \xi} \hat{G}_{\mathrm{cl}}(\xi ; \omega) .
\end{aligned}
$$

Here $d \Omega_{\hat{\mathbf{k}}}$ stands for integration over the directions $\hat{\mathbf{k}}$ with $\Omega_{D}$ being the solid angle in $D$ dimensions. The angle $\theta$ is the angle between $\mathbf{r}$ and $\mathbf{k}$. In step Eq. (A1a), the summation over momenta was converted to an integration over a continuous variable not restricted to the first Brillouin zone, using $D$-dimensional spherical coordinates. This approximation is valid as long as the Fermi wave vector is far away from the Brillouin zone boundaries. In Eq. (A1b), the linear approximation of the dispersion relation $\xi_{k} \approx v_{F}\left(\mathbf{k}-k_{F}\right)$ was employed. Here the Fermi momentum $k_{F}$ is defined as $\xi_{k_{F}}=0$ and the Fermi velocity is the derivative of the dispersion relation evaluated at $k_{F}, v_{F}=d \xi_{k} /\left.d k\right|_{k=k_{F}}$. This approximation is valid as long as the relevant energy scales $\delta \varepsilon$ that enter in the function $\hat{G}_{\mathrm{cl}}$ (such as $\Delta$ and $\omega$ ), as well as in the exponential $\left(v_{F} / r\right)$ satisfy the constraint $|\delta \varepsilon| \ll\left|m^{*}\right| v_{F}^{2} / 2$. Here, $m^{*}$ is the band mass $\left(m^{*}\right)^{-1}=d^{2} \xi_{k} /\left.d k^{2}\right|_{k=k_{F}}$. For a parabolic band, the right-hand side of this inequality is simply the Fermi energy $\varepsilon_{F}$, i.e., the energy difference between the bottom of the dispersion relation at $k=0$ and the Fermi level at $k=k_{F}$. For a linear band $\varepsilon_{F}=v_{F} k_{F}$. So, the linear approximation is valid as long as the energy scales of the problem are much lower than the Fermi energy. In Eq. (A1c), in accordance with the above assumptions, the Fermi energy is taken to infinity. To make the integrals convergent, any high powers of $\xi$ were neglected as well, essentially approximating the DOS per spin component as being flat with energy and equal to its value at the Fermi level:

$$
N_{F}=\frac{\Omega_{D} k_{F}^{D-1}}{(2 \pi)^{D} v_{F}}=\frac{\Omega_{D}}{\lambda_{F}^{D} \varepsilon_{F}},
$$

where $\lambda_{F}=2 \pi / k_{F}$ is the Fermi wavelength. This approximation is in agreement with the above quasicontinuum approximation as long as $k_{F} \ll \pi / a$, with $a$ being the lattice constant. All these approximations are referred to as the wide-band approximation.

Inverting Eq. (15a), we find

$$
\hat{G}_{\mathrm{cl}}(\xi ; \omega)=-\frac{1}{\xi^{2}+|\Delta|^{2}-\omega^{2}}\left(\begin{array}{cc}
\omega+\xi & \Delta \\
\Delta^{*} & \omega-\xi
\end{array}\right),
$$

where the integrals over $\xi$ in Eq. (A1) are performed using the following expressions:

$$
\begin{aligned}
& \int_{-\infty}^{\infty} d \xi \frac{e^{i a \xi}}{\xi^{2}+z}=\frac{\pi}{\sqrt{z}} e^{-|a| \sqrt{z}}, \\
& \int_{-\infty}^{\infty} d \xi \frac{\xi e^{i a \xi}}{\xi^{2}+z}=i \pi \operatorname{sgn}(a) e^{-|a| \sqrt{z}} .
\end{aligned}
$$

These integrals are valid for real $a$ and $\operatorname{Re}[\sqrt{z}]>0$. In Eq. (A4b), it is assumed that $\operatorname{sgn}(0)=0$, in accordance with the Cauchy principal value of the integral. Comparing with Eq. (A3), one can see that $a=r \cos \theta / v_{F}$ and $z=|\Delta|^{2}-\omega^{2}$.

The angular integrals have the following expressions:

$$
\begin{aligned}
\left(\phi_{c}\right)_{D}(r, \omega)= & \int \frac{d \Omega_{\hat{\mathbf{k}}}}{\Omega_{D}} e^{-r\left(\kappa(\omega)|\cos \theta|-i k_{F} \cos \theta\right)} \\
= & \frac{1}{2}\left\{I_{D}\left[\left(\kappa(\omega)+i k_{F}\right) r\right]\right. \\
& \left.+I_{D}\left[\left(\kappa(\omega)-i k_{F}\right) r\right]\right\} \\
\left(\phi_{S}\right)_{D}(r, \omega)= & i \int \frac{d \Omega_{\hat{\mathbf{k}}}}{\Omega_{D}} e^{-r\left(\kappa(\omega)|\cos \theta|-i k_{F} \cos \theta\right)} \operatorname{sgn}(\cos \theta) \\
= & \frac{1}{2 i}\left\{I_{D}\left[\left(\kappa(\omega)+i k_{F}\right) r\right]\right. \\
& \left.-I_{D}\left[\left(\kappa(\omega)-i k_{F}\right) r\right]\right\}, \\
I_{D}(z)= & \int d W_{\hat{\mathbf{k}}} e^{-z \cos \theta} \\
= & \Gamma\left(\frac{D}{2}\right)\left(\frac{z}{2}\right)^{-1+\frac{D}{2}}\left[I_{D / 2-1}(z)-\mathbf{L}_{D / 2-1}(z)\right],
\end{aligned}
$$

where $d W_{\hat{\mathbf{k}}}=\frac{d \Omega_{\hat{\mathbf{k}}} \Theta(\cos \theta)}{\int d \Omega_{\hat{\mathbf{k}}} \Theta(\cos \theta)}$ is a normalized integration measured over only the hyperhemisphere, where $\cos \theta>0$, $\kappa(\omega)=\sqrt{|\Delta|^{2}-\omega^{2}} / v_{F}, I_{\nu}(z)$ is the modified Bessel function of the first kind of order $v$, and $\mathbf{L}_{v}(z)$ is the modified Struve function of order $v$. For $D=1$ and $D=3$, the special function expressions that appear in Eqs. (A5) have an elementary form listed in Table II. Using these results, we obtain Eq. (16). 
TABLE II. Analytic expressions for the functions defined in Eqs. (A5) in $D=1$ and $D=3$ that determine the spatial dependence of the Green's function for the clean system. For $\omega<|\Delta|, \kappa(\omega)$ is real (and positive), which gives exponential decay in $D=1$. In $D=3$, besides the exponentially decaying part there is a constant part in the numerator. For $\omega>|\Delta|, \kappa$ is imaginary which shifts the oscillation period in $D=1$ and in $D=3$. In both cases the overall decay is as $1 / r$ from the $r$ in the denominator.

\begin{tabular}{lccc}
\hline \hline$D$ & $I_{D}(z)$ & $\left(\phi_{c}\right)_{D}(r, \omega)$ & $\left(\phi_{s}\right)_{D}(r, \omega)$ \\
\hline 1 & $e^{-z}$ & $e^{-\kappa(\omega) r} \cos \left(k_{F} r\right)$ & $-e^{-\kappa(\omega) r} \sin \left(k_{F} r\right)$ \\
3 & $\frac{1-e^{-z}}{z}$ & $\frac{-\kappa(\omega)-e^{-\kappa(\omega) r}\left[\kappa(\omega) \cos \left(k_{F} r\right)-k_{F} \sin \left(k_{F} r\right)\right]}{\left[k_{F}^{2}+\kappa^{2}(\omega)\right] r}$ & $\frac{-k_{F}+e^{-\kappa(\omega) r}\left[k_{F} \cos \left(k_{F} r\right)+\kappa(\omega) \sin \left(k_{F} r\right)\right]}{\left[k_{F}^{2}+\kappa^{2}(\omega)\right] r}$ \\
\hline \hline
\end{tabular}

\section{APPENDIX B: $T$-MATRIX EXPRESSION}

The Dyson equation Eq. (12) can be solved by employing a $T$-matrix approach. Namely, the following expression holds:

$$
\begin{aligned}
\breve{G}_{\mathrm{imp}}\left(\mathbf{x}, \mathbf{x}^{\prime} ; \omega\right) & =\breve{G}_{\mathrm{cl}}(\mathbf{x}, \mathbf{0} ; \omega) \cdot \check{T}(\omega) \cdot \breve{G}_{\mathrm{cl}}\left(\mathbf{0}, \mathbf{x}^{\prime} ; \omega\right), \\
\check{T}^{-1}(\omega) & =\frac{1}{U_{m}} \check{P}^{-1}-\check{G}_{\mathrm{cl}}(\mathbf{0}, \mathbf{0} ; \omega) .
\end{aligned}
$$

Having in mind Eqs. (13) and (16a), the solution of Eq. (B1b) for the $T$-matrix is given by

$$
\check{T}(\omega)=\frac{U_{m}}{D_{\mathrm{YSR}}(\omega)}\left[\hat{N}_{T}^{s}(\omega) \otimes \hat{\sigma}_{0}+\hat{N}_{T}^{t}(\omega) \otimes(\mathbf{n} \cdot \hat{\vec{\sigma}})\right],
$$

where the denominator $D_{\mathrm{YSR}}(\omega)$ is given by Eq. (21).

The superscript $s$ refers to the singlet component, and $t$ refers to the triplet component, given by the polarization axis $\mathbf{n}$ of the magnetic impurity atom. The numerators that enter in Eq. (B2), $\hat{N}_{T}^{s}(\omega)$ and $\hat{N}_{T}^{t}(\omega)$, have the following structure in Nambu space:

$$
\begin{aligned}
& \hat{N}_{T}^{s}(\omega)=J_{m} \sqrt{|\Delta|^{2}-\omega^{2}}\left[\left(1+J_{m}^{2}\right) \omega \hat{\tau}_{0}+\left(1-J_{m}^{2}\right) \hat{\Delta}\right] \\
& \hat{N}_{T}^{t}(\omega)=\left[\left(1+J_{m}^{2}\right) \omega^{2}-\left(1-J_{m}^{2}\right)|\Delta|^{2}\right] \hat{\tau}_{0}-2 J_{m}^{2} \omega \hat{\Delta} .
\end{aligned}
$$

\section{APPENDIX C: SPATIAL DEPENDENCE OF BASIS FUNCTIONS}

The pair correlations in Sec. IIID and Sec. IIIC are evaluated at $\mathbf{x}=\mathbf{x}^{\prime}$ in $D=3$. In this section we analyze the spatial dependence of the basis functions Eq. (22) for this choice of coordinates for several values of frequency within and out of the SC gap $|\Delta|$ and for the same choice of SC coherence length $\xi / \lambda_{F}=3$. Because $\psi_{4}$ is odd under the exchange $\mathbf{x} \leftrightarrow \mathbf{x}^{\prime}$ by construction, it is identically zero. At $r=0, \phi_{c}=1$, and $\phi_{s}=0$, so $\psi_{1}=\psi_{2}=1$ and $\psi_{3}=0$ for any $\omega$. All of these functions show oscillatory behavior with a characteristic period equal to $\lambda_{F} / \xi=1 / 3$. In addition, $\psi_{2}$ and $\psi_{3}$ have an additional node at a value smaller than $\lambda_{F} / \xi$. The imaginary part is zero for subgap frequencies and appears only for frequencies higher than the gap. We note there is an overall envelope determined by the squares of the functions $\left(\phi_{c / s}\right)_{3}(r ; \omega)$ given in Table II.

Also, having in mind the nonlocal pair correlations presented in Sec. IIIE, we plot in Fig. 10 the basis functions Eqs. (22) for $\mathbf{x}^{\prime}=\mathbf{0}$ as a function of $r=\mathbf{x}$ for the same choice of frequencies and parameters as in Fig. 9. Similarly to the local case, the imaginary part vanishes for subgap frequencies and different from zero only above the superconducting gap. We note that the overall envelope decay is much slower, because these functions behave as the first powers of $\left(\phi_{c / s}\right)_{3}(r ; \omega)$ given in Table II.
[1] V. L. Berezinskii, ZhETF Pis. Red. 20, 628 (1974) [JETP Lett. 20, 287 (1974)].

[2] A. Balatsky and E. Abrahams, Phys. Rev. B 45, 13125 (1992).

[3] A. M. Black-Schaffer and A. V. Balatsky, Phys. Rev. B 88, 104514 (2013).

[4] J. Linder and A. V. Balatsky, Rev. Mod. Phys. 91, 045005 (2019).

[5] A. J. Leggett, Rev. Mod. Phys. 47, 331 (1975).

[6] J. C. Wheatley, Rev. Mod. Phys. 47, 415 (1975).

[7] E. Abrahams, A. Balatsky, J. R. Schrieffer, and P. B. Allen, Phys. Rev. B 47, 513 (1993).

[8] K. Shigeta, Y. Tanaka, K. Kuroki, S. Onari, and H. Aizawa, Phys. Rev. B 83, 140509(R) (2011).

[9] K. Shigeta, S. Onari, and Y. Tanaka, Phys. Rev. B 85, 224509 (2012).

[10] T. R. Kirkpatrick and D. Belitz, Phys. Rev. Lett. 66, 1533 (1991).

[11] D. Belitz and T. R. Kirkpatrick, Phys. Rev. B 46, 8393 (1992).

[12] D. Belitz and T. R. Kirkpatrick, Rev. Mod. Phys. 66, 261 (1994).
[13] P. Coleman, E. Miranda, and A. Tsvelik, Phys. Rev. Lett. 70, 2960 (1993).

[14] P. Coleman, E. Miranda, and A. Tsvelik, Phys. Rev. B 49, 8955 (1994).

[15] P. Coleman, E. Miranda, and A. Tsvelik, Phys. Rev. Lett. 74, 1653 (1995).

[16] D. L. Cox and A. Zawadowski, Adv. Phys. 47, 599 (1998).

[17] P. O. Sukhachov, V. Juričić, and A. V. Balatsky, Phys. Rev. B 100, 180502(R) (2019).

[18] E. Abrahams, A. Balatsky, D. J. Scalapino, and J. R. Schrieffer, Phys. Rev. B 52, 1271 (1995).

[19] P. O. Sukhachov and A. V. Balatsky, Phys. Rev. B 100, 134516 (2019).

[20] F. S. Bergeret, A. F. Volkov, and K. B. Efetov, Phys. Rev. Lett. 86, 4096 (2001).

[21] A. Kadigrobov, R. I. Shekhter, and M. Jonson, Europhys. Lett. 54, 394 (2001).

[22] F. S. Bergeret, A. F. Volkov, and K. B. Efetov, Rev. Mod. Phys. 77, 1321 (2005). 
[23] J. Linder and J. W. Robinson, Nat. Phys. 11, 307 (2015).

[24] M. Eschrig, Rep. Prog. Phys. 78, 104501 (2015).

[25] A. Di Bernardo, S. Diesch, Y. Gu, J. Linder, G. Divitini, C. Ducati, E. Scheer, M. G. Blamire, and J. W. A. Robinson, Nat. Commun. 6, 8053 (2015).

[26] C. Holmqvist, W. Belzig, and M. Fogelström, Philos. Trans. R. Soc. A 376, 20150229 (2018).

[27] A. V. Balatsky, I. Vekhter, and J.-X. Zhu, Rev. Mod. Phys. 78, 373 (2006).

[28] L. Yu, Acta Phys. Sin. 114, 75 (1965).

[29] H. Shiba, Prog. Theor. Phys. 40, 435 (1968).

[30] A. I. Rusinov, ZhETF Pis. Red. 9, 146 (1969) [JETP Lett. 9, 85 (1969)].

[31] S. Nadj-Perge, I. K. Drozdov, J. Li, H. Chen, S. Jeon, J. Seo, A. H. MacDonald, B. A. Bernevig, and A. Yazdani, Science 346, 602 (2014).

[32] M. Ruby, F. Pientka, Y. Peng, F. von Oppen, B. W. Heinrich, and K. J. Franke, Phys. Rev. Lett. 115, 197204 (2015).

[33] R. Pawlak, M. Kisiel, J. Klinovaja, T. Meier, S. Kawai, T. Glatzel, D. Loss, and E. Meyer, npj Quantum Inf. 2, 16035 (2016).

[34] H. Huang, R. Drost, J. Senkpiel, C. Padurariu, B. Kubala, A. Levy Yeyati, J. C. Cuevas, J. Ankerhold, K. Kern, and C. R. Ast, arXiv:1912.05607 (2019).

[35] T.-P. Choy, J. M. Edge, A. R. Akhmerov, and C. W. J. Beenakker, Phys. Rev. B 84, 195442 (2011).

[36] S. Nadj-Perge, I. K. Drozdov, B. A. Bernevig, and A. Yazdani, Phys. Rev. B 88, 020407(R) (2013).

[37] J. Klinovaja, P. Stano, A. Yazdani, and D. Loss, Phys. Rev. Lett. 111, 186805 (2013).

[38] B. Braunecker and P. Simon, Phys. Rev. Lett. 111, 147202 (2013).

[39] M. M. Vazifeh and M. Franz, Phys. Rev. Lett. 111, 206802 (2013).

[40] F. Pientka, L. I. Glazman, and F. von Oppen, Phys. Rev. B 88, 155420 (2013)

[41] S. Nakosai, Y. Tanaka, and N. Nagaosa, Phys. Rev. B 88, 180503(R) (2013).

[42] K. Pöyhönen, A. Westström, J. Röntynen, and T. Ojanen, Phys. Rev. B 89, 115109 (2014).

[43] Y. Kim, M. Cheng, B. Bauer, R. M. Lutchyn, and S. Das Sarma, Phys. Rev. B 90, 060401(R) (2014).

[44] I. Reis, D. J. J. Marchand, and M. Franz, Phys. Rev. B 90, 085124 (2014).
[45] P. M. R. Brydon, S. Das Sarma, H.-Y. Hui, and J. D. Sau, Phys. Rev. B 91, 064505 (2015).

[46] K. Björnson, S. S. Pershoguba, A. V. Balatsky, and A. M. BlackSchaffer, Phys. Rev. B 92, 214501 (2015).

[47] J. Röntynen and T. Ojanen, Phys. Rev. Lett. 114, 236803 (2015).

[48] J. Li, T. Neupert, Z. Wang, A. H. MacDonald, A. Yazdani, and B. A. Bernevig, Nat. Commun. 7, 12297 (2016).

[49] R. L. R. C. Teixeira, D. Kuzmanovski, A. M. Black-Schaffer, and L. G. G. V. Dias da Silva, Phys. Rev. B 99, 035127 (2019).

[50] B. Sothmann, S. Weiss, M. Governale, and J. König, Phys. Rev. B 90, 220501(R) (2014).

[51] S. Weiss and J. König, Phys. Rev. B 96, 064529 (2017).

[52] C. Triola and A. M. Black-Schaffer, Phys. Rev. B 100, 144511 (2019).

[53] Y. Tanaka and A. A. Golubov, Phys. Rev. Lett. 98, 037003 (2007).

[54] Y. Tanaka, A. A. Golubov, S. Kashiwaya, and M. Ueda, Phys. Rev. Lett. 99, 037005 (2007).

[55] Y. Tanaka, Y. Tanuma, and A. A. Golubov, Phys. Rev. B 76 , 054522 (2007).

[56] A. Yazdani, E. H. da Silva Neto, and P. Aynajian, Annu. Rev. Condens. Matter Phys. 7, 11 (2016).

[57] L. Cornils, A. Kamlapure, L. Zhou, S. Pradhan, A. A. Khajetoorians, J. Fransson, J. Wiebe, and R. Wiesendanger, Phys. Rev. Lett. 119, 197002 (2017).

[58] M. I. Salkola, A. V. Balatsky, and J. R. Schrieffer, Phys. Rev. B 55, 12648 (1997).

[59] Y. Kim, J. Zhang, E. Rossi, and R. M. Lutchyn, Phys. Rev. Lett. 114, 236804 (2015).

[60] S. S. Pershoguba, K. Björnson, A. M. Black-Schaffer, and A. V. Balatsky, Phys. Rev. Lett. 115, 116602 (2015).

[61] K. Björnson, A. V. Balatsky, and A. M. Black-Schaffer, Phys. Rev. B 95, 104521 (2017).

[62] The presence of the magnetic impurity alters the local equaltime pair correlator in its vicinity. Thus, a self-consistent solution for the SC OP would result in a nonuniform SC OP around the impurity. But, since we consider a single impurity immersed in a continuum capable of screening its effects, this nonuniformity is only a second-order correction and not of main concern for the purposes of this work.

[63] T. Bzdušek and R. Hlubina, Philos. Mag. 95, 609 (2015).

[64] V. Perrin, G. C. Ménard, C. Brun, T. Cren, M. Civelli, and P. Simon, arXiv:1912.11241 (2019). 